\title{
An Anisotropic Elastic-plastic Model for the Optimization of a Press Machine's Auxiliary Worktable Plate Thickness
}

\author{
W. Rajhi \\ College of Engineering \\ University of Hail \\ Hail, Kingdom of Saudi \\ Arabia
}

\author{
B. Ayadi \\ College of Engineering \\ University of Hail \\ Hail, Kingdom of Saudi \\ Arabia
}

\author{
A. Alghamdi \\ College of Engineering \\ University of Hail \\ Hail, Kingdom of Saudi \\ Arabia
}

\author{
N. Messaoudene \\ College of Engineering \\ University of Hail \\ Hail, Kingdom of Saudi \\ Arabia
}

\begin{abstract}
In this work an anisotropic elastic-plastic finite element model strongly coupled with ductile damage is applied to determine the suitable thickness of added auxiliary worktable plate to a 100 ton maximum capacity press machine. The major focus of this study is to minimize the amount of stress transmitted to the optional worktable plate and the press machine body while allowing them to withstand plastic deformation and damage during compression testing until final sample fracture. The worktable plates and the press machine body are made of TRIP800 grade steel. AISI 316L stainless steel is chosen as test material for the cylindrical billets. The proposed model is based on a non-associative plasticity theory and the "Hill 1948" quadratic (equivalent) stress norm is considered to describe the large plastic anisotropic flow accounting for mixed isotropic and kinematic hardening with isotropic damage effect. For each material the model uses an experimental data base obtained from a set of tensile tests conducted until the final fracture in three directions, the rolling direction (RD) or 0 , the transverse direction (TD) or 90, and the 45 direction. After several numerical simulations of compression testing using ABAQUS/Explicit FE® software, thanks to the user's developed VUMAT subroutine, varying cylindrical billet diameters and material, worktable plates number and thicknesses and spatial plate configurations the solution of $100 \mathrm{~mm}$ thick worktable plate is selected since in that case the cylinder specimen is totally damaged and the stress state inside the worktable plates and the press machine body remains admissible.
\end{abstract}

Keywords-compression testing; hardening; plastic anisotropy; ductile damage; iIdentification; numerical simulation

\section{INTRODUCTION}

The proposed elastic-plastic model coupled with isotropic ductile damage has been used in several researches to find solutions to a set of problems related especially to the occurrence of the ductile damage, which arises in metal working processes, in order to either delay the damage occurrence during the metal forming processes [1-5] or to stimulate cavitation growth and totally ductile failure in the case of metal cutting processes [5-8]. The extension to the anisotropic damage case of the proposed model in order to simulate the directional effect of the ductile damage in metal forming process has been deeply discussed in $[5,9,10]$. In this work, an isotropic version of the model presented in [9] is used to solve a mechanical engineering problem which consists to determine the suitable thickness of auxiliary worktable plates of a 100ton maximum capacity press machine, their number and their spatial positions in relation to each other, in order to carry out compression testing until final sample fracture while ensuring the protection of the worktable plates and the body of the press machine against plastic deformation and damage (Section II). Section III discusses the deduction of the elasticplastic constitutive equations fully coupled with isotropic damage from the anisotropic damage model case. Numerical aspects are well described in the case of the isotropic damage model in $[1,5,11]$ and extended to the anisotropic damage case in $[5,9,12]$. Section IV is devoted to the identification of the model parameters for the TRIP800 and the AISI 316L grade steels using experimental results from the tensile tests conducted until the final fracture of specimens loaded in three directions i.e., the rolling direction (RD) or 0 , the transverse direction (TD) or 90, and the 45 direction. Thus, the methodology proposed to determine the suitable optional worktable plate thickness is presented and discussed.

\section{PROBLEM FORMULATION}

Press Master (see Figure 1) is a testing press machine which is used in our mechanical laboratory for static three point bending flexural test of steels, aluminum alloys and rock materials under different control parameters. Because the press machine is not equipped with auxiliary worktable, the operating cannot include compression testing. An auxiliary worktable is required to carry out compression testing of different cross sectional area parts. During compression testing the cylindrical specimens are placed between the piston rod and the worktable which is positioned upon the work platform beam. The auxiliary worktable may include one or more metallic plates. These plates and the work platform beam both are made of steel grade that may be assumed to behave elastoplastically with mixed isotropic and kinematic hardening. The TRIP800 steel which constitutes the material of the press 
machine body is selected in this work for the auxiliary worktable plate's material because TRIP steels offer an outstanding combination of strength and ductility being thus suitable for structural and reinforcement parts of complex shape.

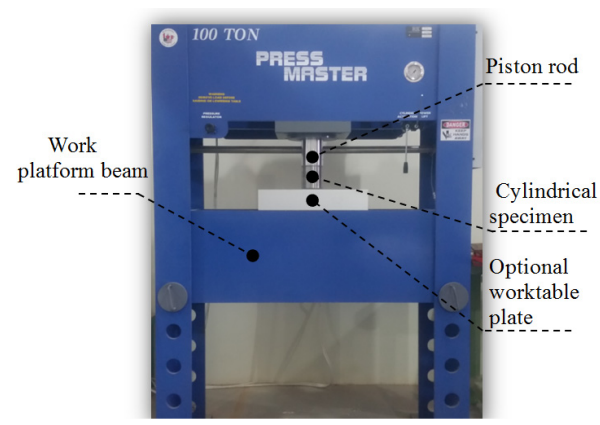

Fig. 1. Illustration of the critical press machine parts.

The typical true stress-strain curves for the TRIP800, resulting from uniaxial tensile tests conducted along the rolling direction (RD) or 0 , the transverse direction (TD) or 90 , and the 45 direction are given in Figure 2. The TRIP800 flat tensile specimen is shown in Figure 3 with all dimensions in millimeters. In Figure 2, one can see that the TRIP800 steel exhibits slight plastic anisotropy with an overall ultimate stress around $1040.0 \mathrm{MPa}$ reached for an overall total strain value of about $24.5 \%$, while the maximum total strain at the final fracture is observed for the rolling direction around $27.0 \%$ i.e., about $26.0 \%$ of plastic strain at fracture. Furthermore, the stress-strain curves obtained for the 45 and (TD) directions have roughly the same elastic-plastic trend before reaching their necking points. The level for which the stress-strain responses bifurcate for each direction is shown in Figure 2 where the 45 stress-strain curve reaches the ultimate stress before that of the (TD) direction. Hence, for many metallic orthotropic sheets, the ductile damage is highly anisotropic even if the anisotropy of the plastic flow is small. Thus, it is worth noting that three tensile tests are performed for each loading direction in order to identify the tensile specimens containing the lower defects density so as to use the corresponding stress-strain curves i.e., revealing the greatest ductility during the model parameters identification.

Present work's purpose is to allow the press machine, within its limit capacity, able to ensure compression testing until final fracture of metallic cylindrical specimens using optional worktable plates made of TRIP800 steel while optimizing the plates' thickness, their number and their configurations according to various spatial arrangements. The problem may be modeled as illustrated in Figure 4. The worktable plates shape is assumed to be square $(400 \mathrm{~mm} * 400 \mathrm{~mm})$ of variable thickness. To achieve the above target, this study aims to predict through a set of numerical simulations of compression testing the limiting diameter of the cylindrical specimens, for a given material, allowing the continuity of the compression process until final fracture while remaining within the press machine capacity range. In addition, the number of the auxiliary plates, their thickness and spatial positions in relation to each other can be optimized so that the maximum values of the equivalent plastic strain and damage reached during the compression process inside the body of the press machine and the worktable plates remain negligible and the stress should have the lower criticality even if it is elastic.

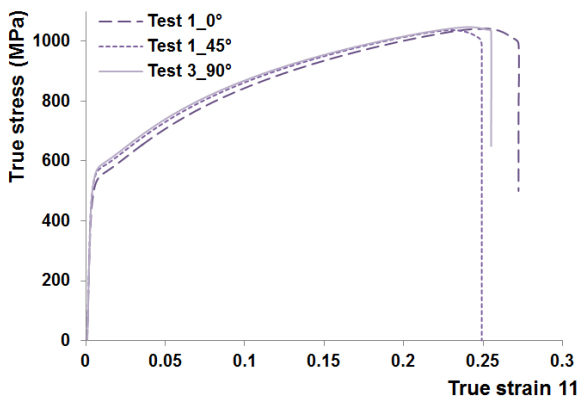

Fig. 2. TRIP800 uniaxial tensile tests conducted along the rolling direction $(\mathrm{RD})$ or 0 , the transverse direction (TD) or 90 , and the 45 direction.

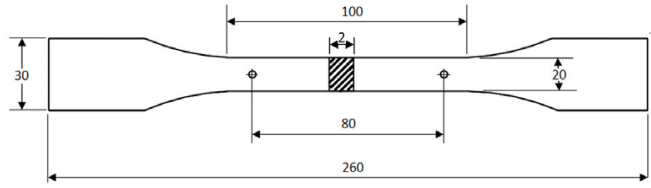

Fig. 3. TRIP800 flat tensile test specimen dimensions.

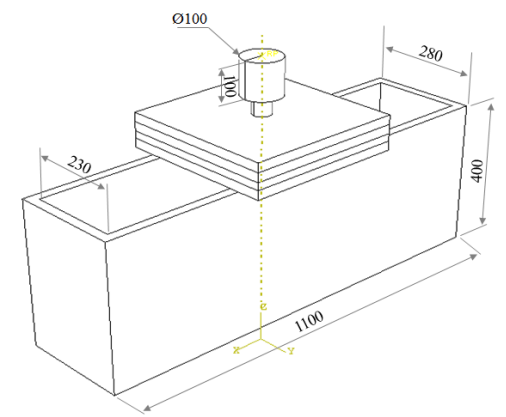

Fig. 4. Schematic representation of the critical press machine parts.

\section{CONSTITUTIVE EQUATIONS}

During compression loading test, the cylindrical sample materials as well as the TRIP800 steel assigned to both the work platform beam and the worktable plates are assumed to behave elastoplastically with mixed isotropic and kinematic hardening according to an anisotropic elastic-plastic model fully coupled to the ductile damage. In this work, only the piston rod will be considered as a rigid part. The proposed model is based on a non-associative plasticity theory and the "Hill 1948" quadratic (equivalent) stress norm is considered to describe the large plastic anisotropic flow accounting for mixed isotropic and kinematic hardening with isotropic damage effect. However, in the context of CDM, when the anisotropic effect of the ductile damage is taken into account (see [10]), a fourthrank damage-effect tensor is used [14-16] to define the effective state variables needed to describe the stress-strain 
behavior accounting for the full effects of the anisotropic ductile damage. In this case, the damage variable may be modeled using a symmetric forth or second rank tensor in order to represent the ductile damage of orthotropic metallic materials. Following the concept of effective stress with the hypothesis of total energy equivalence, the matrix representations of the effective stress and the kinematic stress tensors are given by:

$$
\begin{aligned}
& \underline{\tilde{\sigma}}=\underline{\underline{M}}^{-1}: \underline{\sigma} \\
& \underline{\tilde{X}}=\underline{\underline{M}}^{-1}: \underline{X}
\end{aligned}
$$

where $\underline{\underline{M}}$ is the fourth rank damage effect tensor. In the CDM literature, several forms of damage effect tensor $\underline{\underline{M}}$ are formulated [14]. The effective isotropic hardening stress is given by:

$$
\tilde{R}=\frac{R}{\sqrt{1-\|\underline{\Omega}\|}}
$$

And $\underline{\underline{M}}$ defines an Euclidean norm of the second order damage tensor $\underline{\Omega}$. According to the damage effect tensor forms presented in [14], it is clear that when the ductile damage is assumed to be isotropic the damage effect tensor $\underline{\underline{M}}$ can be written under the following form:

$$
\stackrel{\underline{M}}{\underline{\text { iso }}} \operatorname{diag}\left\{\begin{array}{c}
\frac{1}{f(\Omega)}, \frac{1}{f(\Omega)}, \frac{1}{f(\Omega)}, \\
\frac{1}{f(\Omega)}, \frac{1}{f(\Omega)}, \frac{1}{f(\Omega)}
\end{array}\right\}
$$

where $\Omega$ is the isotropic damage variable and $f(\Omega)$ is a degreasing function of $\Omega$ called the damage effect function. To make the link between the constitutive equations of the anisotropic damage model and those of the isotropic damage case, the following damage effect function is chosen to describe the stress-strain behavior accounting for the full effects of the isotropic ductile damage:

$$
f(\Omega)=\sqrt{1-\Omega}
$$

The constitutive equations for the isotropic damage case may be deduced readily from those of the anisotropic case by substituting the damage effect tensor $\underline{\underline{M}}$ by the isotropic damage effect tensor given by:

$$
{ }^{i s o} \underline{\underline{M}}=\sqrt{1-\Omega} \stackrel{1}{=}
$$

Where 1 is the fourth order unit tensor. Thus, in the isotropic damage case, the Euclidean norm $\|\underline{\Omega}\|$ of the second order damage tensor transforms to the scalar damage variable $\Omega$. In this work the plastic anisotropy is taken into account via the fourth rank symmetric and positive definite operator
$\underline{\underline{H}}$ which defines the anisotropy of plastic flow. It is expressed in terms of Hill's anisotropic parameters $F, G, H, L, M$ and $N$ :

$$
\underline{\underline{H}}=\left[\begin{array}{cccccc}
G+H & & & & & \text { Sym. } \\
-H & H+F & & & & \\
-G & -F & F+G & & & \\
0 & 0 & 0 & 2 L & & \\
0 & 0 & 0 & 0 & 2 M & \\
0 & 0 & 0 & 0 & 0 & 2 N
\end{array}\right]
$$

Using the non-associative framework, the anisotropic plastic flow is modeled using a yield function $f_{P}$ :

$$
f^{p}(\underline{\sigma}, \underline{X}, R ; \underline{\Omega})=\|\underline{\tilde{\sigma}}-\underline{\tilde{X}}\|_{H}-\tilde{R}-\sigma_{y}
$$

The parameter $\sigma_{y}$ is the initial size of the plastic yield surface. The norm $\|\underline{\tilde{\sigma}}-\underline{\tilde{X}}\|_{H}$ defines the "Hill 1948" quadratic equivalent stress in the effective stress space. The fully coupled anisotropic plasticity-isotropic damage model constitutive equations accounting for the kinematic and isotropic hardening are given by:

$$
\begin{aligned}
& \underline{\sigma}=E(1-\Omega) \underline{\varepsilon}^{e} \\
& \underline{X}=C(1-\Omega) \underline{\alpha} \\
& R=Q(1-\Omega) r
\end{aligned}
$$

where $E, C$ and $Q$ are the elasticity, kinematic and isotropic hardening modules. The application of the generalized normality rule leads to the evolution relations for plastic flow with hardening and anisotropic damage effect:

$$
\begin{aligned}
& \dot{\varepsilon}^{p}=\dot{\lambda} \underline{n} \\
& \dot{r}=\frac{\dot{\lambda}}{\sqrt{1-\Omega}}(1-b \tilde{r}) \\
& \dot{\dot{\alpha}}=\underline{\varepsilon}-a \dot{\lambda} \underline{\alpha}
\end{aligned}
$$

where $\underline{n}$ represents the outward unit normal to the plastic yield surface in the damaged state. The material constants $a$ and $b$ are the non-linearity parameters for kinematic and isotropic hardening respectively. $\dot{\lambda}$ is the plastic (Lagrange) multiplier. The effect of the damage is crucially governed by (6) and (15):

$$
\dot{\Omega}=\frac{\dot{\lambda}}{(1-\Omega)^{\beta}}\left(\frac{Y-Y_{0}}{S}\right)^{s}
$$

where $Y$ is the total energy release rate density tensor. In conclusion, noting that both the anisotropic and isotropic damage models have the same number of material parameters which are the elastic proprieties $E$ and $v$ to describe the isotropic elastic behavior, eleven plastic parameters $\sigma_{y}, F, G, H$, $L, M, N, C, Q, a$ and $b$ for the plastic anisotropy and four 
damage parameters $S, s, \beta$ and $Y_{0}$ to control the damage evolution. Finally, the related numerical aspects are deeply described in $[1,5,11]$.

\section{RESULTS}

This section is devoted to the numerical simulation of compression testing of cylinder specimens made of different materials according to the schematic representation illustrated in Figures 3-4. The identification of the elastic-plastic and damage parameters of the isotropic damage model presented above are required to perform the numerical simulation of compression testing. Two grade steel materials are considered for each compression testing simulation. The TRIP800 steel for the press machine parts (the work platform beam and the worktable plates) and the AISI 316L stainless steel assigned beforehand to the cylindrical specimen. The identification procedure is based on two main steps. The first step consists on the determination of the elastic-plastic parameters through a set of uncoupled (without damage effect) tensile test calculations under Abaqus/Explicit ${ }^{\circledR}$ using the user subroutine VUMAT while respecting the specimen geometry (see Figure 4). The tensile specimen given in Figure 3 is meshed using hexahedral tri-linear elements $(C 3 D 8 R)$ with a meshing refinement (minimum mesh size of $1.0 \mathrm{~mm}^{3}$ ) located on the central region or gage length of the specimen. The numerical tensile test was carried out with a constant velocity of $3.0 \mathrm{~mm} / \mathrm{s}$. Anisotropic parameters $G$ and $H$ and the set of hardening parameters are varied in order to get the best fit between the numerical forcedisplacement curve and the one obtained experimentally along the rolling direction while respecting two following conditions: 1) $G+H=1$ and 2) the retained plasticity parameters should provide a numerical response comparable with experience, up to a plastic strain range between $15 \%$ and $20 \%$, from which the two responses bifurcate due to the emphasized effect of ductile void growth on experimental response. The TRIP800 (TD) 90 and 45 force-displacement experimental responses are explored later to determine the remaining parameters $F$ and $N$ respectively taking into account that $\sigma_{90}=\sigma_{y} / \sqrt{F+H}$ and $\sigma_{45}=2 \sigma_{y} / \sqrt{F+g+2 N}$. In the second identification step, fully coupled calculations are performed taking into account the damage effect in order to recover the optimum damage parameters which provide the best fit between the end of numerical and experimental forcedisplacement curves. In conclusion, the same VUMAT is used with a flag parameter (NCD) allowing to perform an uncoupled analysis when $\mathrm{NCD}=0$ and a fully coupled analysis when $\mathrm{NCD}=1$. The superposed experimental, numerical uncoupled and fully coupled force-displacement curves for the three loading directions RD, 45 and TD for the TRIP800 steel are not presented here (see [9] for more details concerning the AISI 316L material parameters identification) and we limit ourselves only to report in Table 1 the best values of the TRIP800 and AISI 316L steels parameters resulting from the identification procedure described above. The parts shown in Figure 5, excluding the piston rod, are meshed using hexahedral trilinear elements $(C 3 D 8 R)$ with an overall meshing size of about $2.0 \mathrm{~mm}^{3}$ for the $50 \mathrm{~mm}$ length cylinder specimen, $7 \mathrm{~mm}^{3}$ for the $100 \mathrm{~mm}$ thick worktable plate and $20 \mathrm{~mm}^{3}$ for the encastred work platform beam. The piston rod is considered as a rigid part meshed using bilinear rigid quadrilateral elements (R3D4) with meshing size of about $5.0 \mathrm{~mm}^{3}$. If a modification of the meshing size defined during the fully coupled analysis of the identification procedure is intended, before performing the compression testing simulations with the new meshing size, the damage parameters values given in Table I particularly the parameter $S$ should be adjusted by supplementary set of identification calculations for each material as the proposed model is written in local approach where the damage parameters depend strongly on the meshing size [18]. The numerical compression testing was carried out with a constant piston velocity of $100.0 \mathrm{~mm} / \mathrm{s}$. The friction coefficient defined between the different parts is about 0.3 . In order to reduce the CPU time in the followed dynamic explicit algorithm, a mass scaling value of $10^{-6}$ is defined through all the compression testing simulations. According to Figure $6,36 \mathrm{~mm}$ is the limiting diameter value of the AISI $316 \mathrm{~L}$ cylinder specimens which the press machine can totally damage without exceeding its maximum loading capacity range. Otherwise, beyond this limiting diameter, the loading amount provided by the press machine is unable to ensure the total failure as revealed by the numerical simulation of the compression testing of the AISI $316 \mathrm{~L}$ cylinder specimens given by Figure 6, where the damage doesn't exceed $5 \%$ and $10 \%$ for 44 and $40 \mathrm{~mm}$ cylinder diameters respectively when the loading reaches the maximum capacity force of the press machine.

TABLE I. TRIP800 AND AISI 316L MODEL PARAMETERS

\begin{tabular}{|c|c|c|}
\hline \multirow{2}{*}{$\begin{array}{c}\text { Model } \\
\text { Parameters }\end{array}$} & \multicolumn{2}{|c|}{ Grade Steel } \\
\cline { 2 - 3 } TRIP800 & AISI 316L [9] \\
\hline E & $195000 \mathrm{MPa}$ & $200000 \mathrm{MPa}$ \\
\hline$v$ & 0.3 & 0.3 \\
\hline \multicolumn{3}{|c|}{ Anisotropic Plasticity } \\
\hline$\sigma y$ & $406 \mathrm{MPa}$ & $225 \mathrm{MPa}$ \\
\hline $\mathrm{F}$ & 0.45 & 0.8 \\
\hline $\mathrm{G}$ & 0.47 & 0.76 \\
\hline $\mathrm{H}$ & 0.53 & 0.24 \\
\hline $\mathrm{L}$ & 1.5 & 1.5 \\
\hline $\mathrm{M}$ & 1.5 & 1.5 \\
\hline $\mathrm{N}$ & 1.45 & 1.55 \\
\hline $\mathrm{C}$ & $49000 \mathrm{MPa}$ & $15000 \mathrm{MPa}$ \\
\hline $\mathrm{Q}$ & $4000 \mathrm{MPa}$ & $2000 \mathrm{MPa}$ \\
\hline $\mathrm{a}$ & 380 & 300 \\
\hline $\mathrm{b}$ & 4.2 & 0.6 \\
\hline \multicolumn{3}{|c|}{} \\
\hline $\mathrm{S}$ & Damage & $140 \mathrm{MPa}$ \\
\hline $\mathrm{s}$ & $102 \mathrm{MPa}$ & 1 \\
\hline$\beta$ & 1 & 0 \\
\hline $\mathrm{Y} 0$ & 1.5 & \\
\hline \multicolumn{3}{|c|}{} \\
\hline
\end{tabular}

The first set of compression testing simulations checks the suitable AISI 316L cylinder specimen diameter which the press machine can deform until total failure occurs without exceeding the capacity specified by the manufacturer. In Figure 5 initial geometries, mesh and boundary conditions of the compression testing simulation are schematized. The worktable is assumed to be made from one plate $100 \mathrm{~mm}$ thick. The distribution of the Von Mises stress, the damage and the equivalent plastic strain inside the $36 \mathrm{~mm}$ diameter cylinder at the end of the compression testing process is shown in Figure 7. The mechanical field distribution within the worktable plate 
and the body of the press machine analysis will be discussed later. Furthermore, the compression testing simulation was successfully accomplished as the maximum values of the damage and the equivalent plastic strain were located at the center of the AISI $316 \mathrm{~L}$ cylinder correctly at the intersection of the two well-known shear bands. This result is marked by the fast drop of the force-displacement curve (not presented here) at the end of the compression testing. According to Figure 7(a), the maximum VOM stress obtained at the end of the compression process within the cylinder $\sigma_{\text {end }}\left(\sigma_{\text {end }}=860 \mathrm{MPa}\right)$ is considerably greater than the ultimate stress of the AISI $316 \mathrm{~L}$ [9] as significant dissimilarity between the tensile and the compression behaviors at large deformations is often expected in ductile materials.

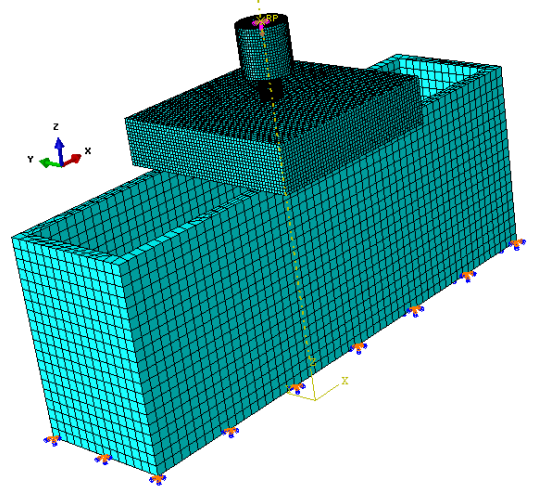

Fig. 5. Geometry, boundary conditions, and meshing of the parts.

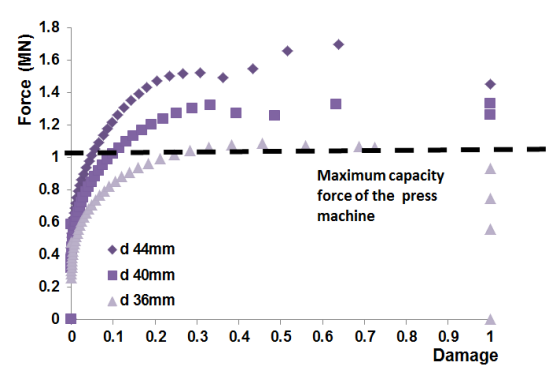

Fig. 6. Numerical force-damage curves of the AISI $316 \mathrm{~L}$ compression testing obtained for different diameters

A second set of compression testing simulations was carried out altering TRIP800 worktable plate's number, plate thickness and configuration according to various spatial arrangements of plates from one calculation to another until obtaining the optimal configuration which ensures the protection of the worktable plates and the body of the press machine against plastic deformation and damage. Furthermore, the solutions exhibiting low criticality were collected. It is worth noting that the appropriate configuration means the numerical solution allowing the compression testing until total failure of the AISI $316 \mathrm{~L}$ cylindrical specimen without exceeding the press machine capacity and giving the lowest elastic stress value inside both the TRIP800 worktable plates and the press machine body. After several numerical simulations of compression testing, the solution of $100 \mathrm{~mm}$ maximum thickness is selected for a well-defined number of worktable plates. Accordingly, eleven configurations based on how worktable plates with different number and thicknesses are arranged spatially, were analyzed. In proposed configurations, the plates are positioned from the piston rod to the work platform beam across the plates thickness direction according to the rank reported in Table II.

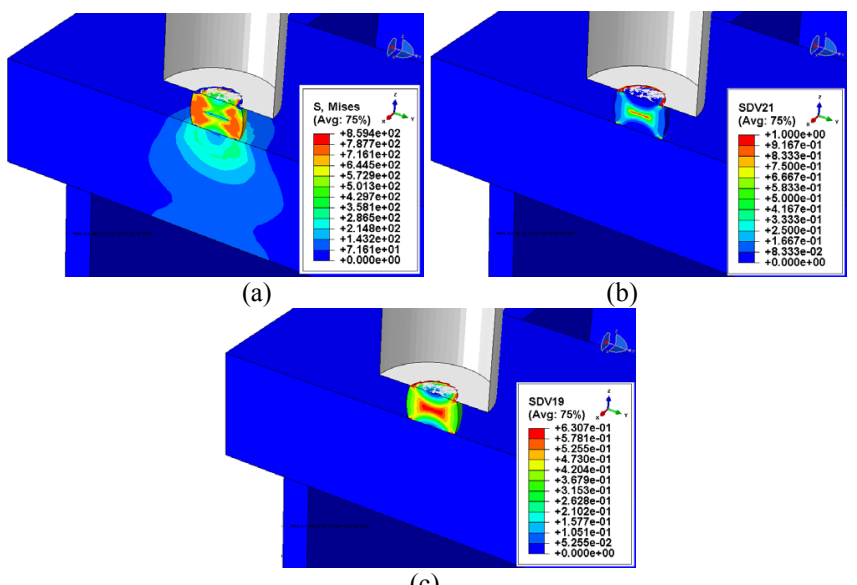

(c)

Fig. 7. (a) Distribution of the Von Mises stress, (b) the damage and (c) the equivalent plastic strain, at the end of the compression testing of $36 \mathrm{~mm}$ diameter cylindrical AISI 316L billet.

TABLE II. CONFIGURATIONS AND RELATED MAXIMUM VOM STRESS STATES OBTAINED DURING COMPRESSION TESTING OF THE AISI 316L SAMPLES

\begin{tabular}{|c|c|c|c|}
\hline \multicolumn{2}{|c|}{ Configuration } & $\begin{array}{c}\text { VOM Stress } \\
\text { Maxi in } \\
\text { plates } \boldsymbol{\sigma}^{\boldsymbol{P}} \\
\text { (MPa) }\end{array}$ & $\begin{array}{c}\text { VOM Stress Maxi } \\
\text { in machine body } \boldsymbol{\sigma}^{\boldsymbol{B}} \\
\text { (MPa) }\end{array}$ \\
\hline A & 1 plate of 50mm & 462 & 200 \\
\hline B & 1 plate of 75mm & 429 & 110 \\
\hline C & 3 plates of 25mm & 556 & 319 \\
\hline D & 2 plates of 37.5mm & 463 & 208 \\
\hline E & 2 plates of 50mm & 430 & 129 \\
\hline F & 1 plate of $100 \mathrm{~mm}$ & 421 & 81 \\
\hline G & $\begin{array}{c}2 \text { plates of } \\
25 \rightarrow 50 \mathrm{~mm}\end{array}$ & 481 & 181 \\
\hline H & $\begin{array}{c}2 \text { plates of } \\
50 \rightarrow 25 \mathrm{~mm}\end{array}$ & 439 & 156 \\
\hline I & $\begin{array}{c}3 \text { plates of } \\
25 \rightarrow 50 \rightarrow 25 \mathrm{~mm}\end{array}$ & 478 & 152 \\
\hline J & $\begin{array}{c}3 \text { plates of } \\
50 \rightarrow 25 \rightarrow 25 \mathrm{~mm}\end{array}$ & 445 & 99 \\
\hline K & $\begin{array}{c}2 \text { plates of } \\
75 \rightarrow 25 \mathrm{~mm}\end{array}$ & 432 & \\
\hline
\end{tabular}

Maximum VOM stress values reported in Table II are reached immediately before the total rupture of the first meshing element generally located at the specimen center. Configurations reported in Table II are judged to be of low criticality as the maximum VOM stress within the body of the press machine is less than the yield stress of the TRIP800 steel. The above configurations are chosen among harshest stress state configurations causing a significant problem within the worktable plates and the press machine body. In fact, as illustrated in Figure $8(\mathrm{a})$, the use of two $20 \mathrm{~mm}$ thick plates 
leads to the excessive bending distortion of the TRIP800 worktable plates before the total fracture of the AISI 316L specimen occurs. Likewise, as illustrated in Figure 8(b), high stress i.e., $98 \%$ of the TRIP 800 yield stress, is detected within the press machine body with a slight bending distortion of the inferior plates owing to the use of five $20 \mathrm{~mm}$ thick plates. The results reported in Table II prove the TRIP800 steel's validity while giving the worktable plates excellent combination of strength and ductility under extreme loading conditions since almost the maximum VOM stress values inside the plates $\sigma \mathrm{P}$ for various configurations are not very far from the yield stress value of the TRIP800 steel. The slight decrease of the billet diameter carries the greater part of the configurations below the elastic barrier. Nevertheless, the maximum VOM stress values within the press machine body $\sigma \mathrm{B}$ remain below the elastic limit for all configurations. According to the configurations sets $(\mathrm{B}, \mathrm{C}, \mathrm{D})$ and $(\mathrm{F}, \mathrm{K}, \mathrm{I}$ or $\mathrm{J})$, the higher the number of plates, the higher the maximum VOM stress within the plates and the press machine body. The couple of values defined by the gaps between $\sigma_{\text {end }}$ and the maximum VOM stress within both the worktable plates and the press machine body $\left(\sigma_{e n d}-\sigma^{P}\right.$, $\left.\sigma_{\text {end }}-\sigma^{B}\right)$ is used as criterion for the selection of the suitable configuration. Accordingly, the $\mathrm{F}$ and $\mathrm{K}$ configuration provides the maximum pair gap values of about $(440,780 \mathrm{MPa})$ and $(428,761 \mathrm{MPa})$ for the plates and the press machine body respectively and may be chosen as the appropriate configurations.

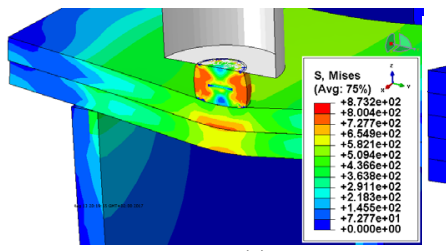

(a)

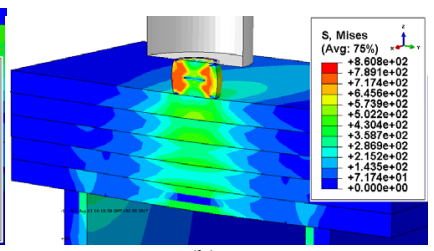

(b)
Fig. 8. (a) Excessive bending distortion of two $20 \mathrm{~mm}$ thick plates, (b) high stress in the machine press body due to the use of five $20 \mathrm{~mm}$ thick plates

\section{CONCLUSION}

In this work, a mechanical engineering problem related to the optimization of the press machine optional worktable thickness was solved using an anisotropic elastic-plastic finite element model strongly coupled with ductile damage. The numerical simulation of compression testing of different materials varying worktable plates' thickness, their number and their spatial positions in relation to each other is crucial in order to find the best solution allowing the worktable plates and the press machine body to withstand against plastic deformation and damage. The use of $100 \mathrm{~mm}$ worktable plate reduces greatly the amount of stress transmitted to the press machine parts. Eventually, the above solution will be considered practically to perform compression testing of cylindrical samples made of any material.

\section{REFERENCES}

[1] K. Saanouni, J. F. Mariage, A. Cherouat, P. Lestriez, "Numerical prediction of discontinuous central bursting in axisymmetric forward extrusion by continuum damage mechanics", Computers \& structures, Vol. 82, No. 27, pp. 2309-2332, 2004

[2] M. Khelifa, H. Badreddine, A. Daoud, M. A. Gahbich, K. Saanouni, A. Cherouat, A. Dogui, "Effect of anisotropic plastic flow on the ductile damage evolution in hydrobulging test of thin sheet metal", International Journal of Forming Processes, Vol. 8, No. 2, pp. 271-289, 2005

[3] H. Badreddine, C. Labergere, K. Saanouni, W. Rajhi, A. Rassineux, D. Kircher, "FE elastoplastic damage model with $2 \mathrm{D}$ adaptive remeshing procedure for fracture prediction in metal forming simulation", International Journal of Material Forming, Vol. 1, pp. 109-112, 2008

[4] C. Labergère, K. Saanouni, P. Lestriez, "Numerical design of extrusion process using finite thermo-elastoviscoplasticity with damage. Prediction of chevron shaped cracks", Key Engineering Materials, Vol. 424, pp. 265-272, 2010

[5] K. Saanouni, Damage Mechanics in Metal Forming: Advanced Modeling and Numerical Simulation,Wiley, 2012

[6] M. Issa, K. Saanouni, C. Labergere, "Prediction of serrated chip formation in orthogonal metal cutting by advanced adaptive 2D numerical methodology", International Journal of Machining and Machinability of Materials, Vol. 9, No. 3, pp. 295-315, 2011

[7] K. Saanouni, N. Belamri, P. Autesserre, "3D numerical simulation of anisotropic thin sheet metal slitting process using fully coupled constitutive equations including ductile damage", International Journal of Material Forming, Vol. 2, No. 1, pp. 535-538, 2009

[8] C. Labergère, A. A. Rassineux, K. Saanouni, "2D adaptive mesh methodology for the simulation of metal forming processes with damage", International Journal of Material Forming, Vol. 4, No. 3, pp. 317-328, 2011

[9] W. Rajhi, K. Saanouni, H. Sidhom, "Anisotropic ductile damage fully coupled with anisotropic plastic flow: Modeling, experimental validation, and application to metal forming simulation", International Journal of Damage Mechanics, Vol. 23, No. 8, pp. 1211-1256, 2014

[10] K. Saanouni, J. L. Chaboche, "Computational damage mechanics: Application to metal forming simulation", Comprehensive Structural Integrity.Vol. 3, pp. 321-376, 2003

[11] H. Badreddine, K. Saanouni, A. Dogui, "On non-associative anisotropic finite plasticity fully coupled with isotropic ductile damage for metal forming", International Journal of Plasticity, Vol. 26, No. 11, pp. 15411575,2010

[12] H. Badreddine, K. Saanouni, T. D. Nguyen, "Damage anisotropy and its effect on the plastic anisotropy evolution under finite strains", International Journal of Solids and Structures, Vol. 63, pp. 11-31, 2015

[13] K. Saanouni, A. Cherouat, Y. Hammi, "Numerical aspects of finite elastoplasticity with isotropic ductile damage for metal forming", Revue Européenne des Elements Finis, Vol. 10, No. 2-4, pp. 327-351, 2001

[14] C. L. Chow, T. J. Lu, “A normative representation of stress and strain for continuum damage mechanics", Theoretical and Applied Fracture Mechanics, Vol. 12, pp.161-187, 1989

[15] C. L. Chow, J. Wang, "An anisotropic theory of continuum damage mechanics for ductile fracture", Engineering Fracture Mechanics, Vol. 27, pp. 547- 558, 1987

[16] C. L. Chow, J. Wang, "Ductile fracture characterization with an anisotropic continuum damage theory", Engineering Fracture Mechanics Vol. 30, pp. 547-563, 1988

[17] J. Lemaitre, R. Desmorat, M. Sauzaya, "Anisotropic damage law of evolution", European Journal of Mechanics, Vol. 19, pp. 187-208, 2000

[18] K. Saanouni, M. Hamed, "Micromorphic approach of finite gradientelastoplasticity fully coupled with ductile damage: Formulation and computational approaches", International Journal of Solids and Structures, Vol. 50, pp. 2289-2309, 2013 